\title{
The role of C-reactive protein ratio in predicting mortality in patients with Fournier gangrene
}

\author{
Ismail Cem Eray ${ }^{1}$, Kubilay Dalci ${ }^{1}$, Serdar Gumus ${ }^{2}$, Orcun Yalav ${ }^{1}$, Ahmet Gokhan Saritas ${ }^{1}$, Asli Boz ${ }^{3}$, \\ Ahmet Rencuzogullari ${ }^{1}$
}

${ }^{1}$ Division of Colorectal Surgery, Department of General Surgery, Çukurova University Faculty of Medicine, Adana; Departments of ${ }^{2}$ Surgical Oncology and ${ }^{3}$ Medical Education, Çukurova University Faculty of Medicine, Adana, Turkey

Purpose: This study aimed to determine the C-reactive protein (CRP) ratio for the survival of patients with Fournier gangrene (FG).

Methods: Fifty-two patients with FG between January 2011 and September 2018 were retrospectively analyzed. Data on clinical presentation, Fournier Gangrene Severity Index (FGSI), CRP ratio, management, and outcome were analyzed. The CRP ratio was calculated as preoperative CRP/postoperative CRP value that measured 48 hours after surgical intervention. Possible alternative cutoff points for the FGSI and CRP were determined by receiver operating characteristic (ROC) analyses. The risk factors related to the prognosis were evaluated by univariate and multivariable logistic regression analyses.

Results: The mean CRP ratios were $6.7 \pm 6.6$ in the survivor group and $1.2 \pm 0.8$ in the non-survivor group $(\mathrm{P}=0.001)$. FGSI was significantly higher in the non-survivor group compared to survivor group $(8.5 \pm 2.5 \mathrm{vs.} 3.5 \pm 2.2, \mathrm{P}=0.001)$. There was a negative correlation between FGSI and CRP ratio $(\mathrm{r}=-0.51)$. ROC analysis determined the cutoff value as 1.78 for CRP (sensitivity, $86 \%$; specificity, $82 \%$; AUC, 0.90 ) to predict death. The incidence of death for patients with CRP ratio of $\leq 1.78$ increased 26.7 fold for those with CRP ratio of $>1.78$ (95\% confidence interval [CI], 4.8-146.5; P =0.001). In the multivariable logistic regression model, CRP ratio (odds ratio [OR], 10.3; 95\% CI, 1.5-72.2; P=0.019) and FGSI (OR, 17.8; 95\% CI, 2.6$121.1 ; \mathrm{P}=0.003$ ) were independent risk factors for death.

Conclusion: The CRP ratio is a simple method to use to predict mortality in FG.

Keywords: Fournier gangrene; C-reactive protein; Mortality

\section{INTRODUCTION}

Fournier gangrene (FG) is the necrotizing soft tissue infection of the perineum which spreads rapidly along the deep fascial planes cause poor prognosis such as mortality $[1,2]$. Considering the increasing prevalence of FG, conceivably due to advances in the

Received: Sep 25, 2021 - Revised: Dec 7, 2021 - Accepted: Dec 13, 2021 Correspondence to: Serdar Gumus, M.D., FTBS

Department of Surgical Oncology, Faculty of Medicine, Çukurova University, Sarıcam 01330, Adana, Turkey

Tel: +90-530-611-70-03, Fax: +90-322-338-69-45

E-mail: seredargumus@hotmail.com

ORCID: https://orcid.org/0000-0001-7629-9369

(C) 2022 The Korean Society of Coloproctology

This is an open-access article distributed under the terms of the Creative Commons Attribution NonCommercial License (https://creativecommons.org/licenses/by-nc/4.0) which permits unrestricted noncommercial use, distribution, and reproduction in any medium, provided the original work is properly cited. treatment of metabolic and oncological diseases leading to form aging populations and high levels of morbidity, efforts to identify patients at the highest risk of mortality have paramount importance [3].

The disease is still associated with high mortality nowadays, and its mortality rate ranges from $20 \%$ to $30 \%$ in the current literature [4]. Because of these high mortality rates, scoring systems that can predict mortality early are important for patient management and treatment needs. That is why different scoring systems have been developed for predicting mortality due to FG. To develop some prognostic parameters and follow-up these patients with these parameters may help coping-handling negative process of these patients.

In 1995, Laor et al. [5] described the Fournier Gangrene Severe Index (FGSI), which used temperature, heart rate, respiratory rate, serum potassium and sodium, creatinine, bicarbonate levels, 
hematocrit, and white blood count. In 2010, Yilmazlar et al. [6] added the patient's disease dissemination status and age to FGSI and described this new scoring as the Uludag FGSI (UFGSI). FGSI and UFGSI scoring systems most widely used methods for predicting mortality due to FG have shown acceptably high sensitivity and specificity. However, both scoring systems are quite complex. Due to this complexity, their implementation is not practical in the clinical setting. Therefore, there is a need for simpler and more practical scoring systems in clinical practice. The scoring described by Lin et al. [7] is an example of the simple scoring system called simplified FGSI using fewer parameters but without loss of sensitivity and specificity. If a reliable, simplified scoring system can be developed that is easier for clinicians to calculate, the likelihood of clinical use increases.

As dynamic analysis of biological variables seems more relevant than isolated parameters predicting outcomes in critically ill patients, we hypothesized that an effective decrease in C-reactive protein (CRP) concentrations after surgical debridement predicts improved survival in FG patients. CRP, an acute-phase protein, has been extensively addressed in critically ill patients in terms of mortality. Its feature reflects the intensity of the ongoing inflammatory stimulus [8]. To determine the reliability of the CRP ratio as a predictor variable, we compared it with the FGSI scoring index. The main endpoint of our study was to determine whether CRP levels of pre- and postsurgical debridement is a predictive factor in mortality. CRP is one of the best tests to show inflammation caused by infection, trauma, inflammatory rheumatic, and malignant diseases. It begins to rise in a short time after inflammation and reaches a maximum in 48 hours [8]. The half-life of CRP is about 19 hours. Therefore, when the inflammatory cause disappears in a person with high CRP levels, the CRP level is expected to decrease the next day significantly [9]. The decrease in CRP levels after aggressive debridement, which is the basis of FG treatment, may indicate that the focus causing inflammation is effectively eliminated $[8,9]$. Therefore, in the present study, we compared the CRP levels at the time of diagnosis with the values 48 hours after debridement.

In this study, we aimed to evaluate clinical course, management, and risk factors with a particular emphasis on the prognostic significance and also to evaluate the role of reduction in CRP as a prognostic factor for patients with FG and present our follow-up and treatment experience over 6 years for these patients.

\section{METHODS}

A retrospective cross-sectional review was conducted of all patients presenting to Division of Colorectal Surgery, Çukurova University Faculty of Medicine (a tertiary-care center in Adana, Turkey) with FG between January 2011 and September 2018. Data were obtained from our prospectively maintained database, and the study was approved by the Institutional Review Board of Çukurova University Faculty of Medicine (No. 113/date: July 9,
2021). Written informed consent was obtained from all patients upon admission to our hospital that their clinical data could be used in future studies. It has been performed by the ethical standards laid down in the 1964 Declaration of Helsinki. Patients undergoing debridement by the urology department for their FG strictly localized to genitalia were excluded $(n=18)$.

All patients diagnosed with FG received supportive intravenous fluid resuscitation and broad-spectrum antibiotic treatment, which was subsequently modified until a resistogram was available. Immediate and aggressive debridement consisting of all necrotic skin, subcutaneous tissue, fascia, and muscle resection was performed until viable tissue margins were identified by colorectal surgeons (ICE and AR). The decision on whether to perform a proximal diverting stoma was based on the surgeon's preference, which was mainly determined by the status of the anal sphincter complex and immunocompromised state of the patients. For patients with complex wounds, vacuum-assisted closure (VAC) devices were employed after first or second debridement to decrease fecal contamination risk regardless of anal sphincter injury. VAC dressings were changed every 72 hours, whereas conventional wound dressings were repeated daily by the well-coordinated team, including the surgeon, stoma and wound therapy nurse. Patients who are not considered a candidate for diverting stoma and those within some degree of anal sphincter injury were managed with a bowel management catheter (BMC) to decrease fecal contamination of the wound (Fig. 1). Once the wound is completely healed decision is made to close with tertiary intention or reconstructive procedures, including the split-thickness skin graft, full-thickness skin graft, local advancement flap, fasciocutaneous flap, muscle flap, and myocutaneous flap. BMC is continued postoperatively to decrease the risk of wound infection.

The following patient and surgery-related parameters were analyzed; age, sex, comorbid factors (diabetes mellitus [DM], cardiovascular disease, etc.), the number of debridement performed, amount of blood transfusion (unit), requirement of fecal diversion, length of hospital stay, bacteriological results of detectable species in wound cultures, the use of negative pressure wound therapy and/or BMC.

The patients were divided into 2 groups; those who died during treatment and those alive. Their clinical features were compared between the 2 groups.

In addition, FGSI was calculated in our study. To calculate FGSI, the following variables at admission were abstracted; temperature, heart rate, respiratory rate, serum potassium, serum sodium, serum creatinine, hematocrit, leukocyte count, and serum bicarbonate concentrations $[5,6]$. As a marker reflecting the trend in concentration, the CRP ratio was calculated. The CRP ratio was calculated by dividing CRP level before debridement by the CRP level measured 48 hours after surgical intervention. The CRP ratio means preoperative $\mathrm{CRP} /$ postoperative $\mathrm{CRP}$. 

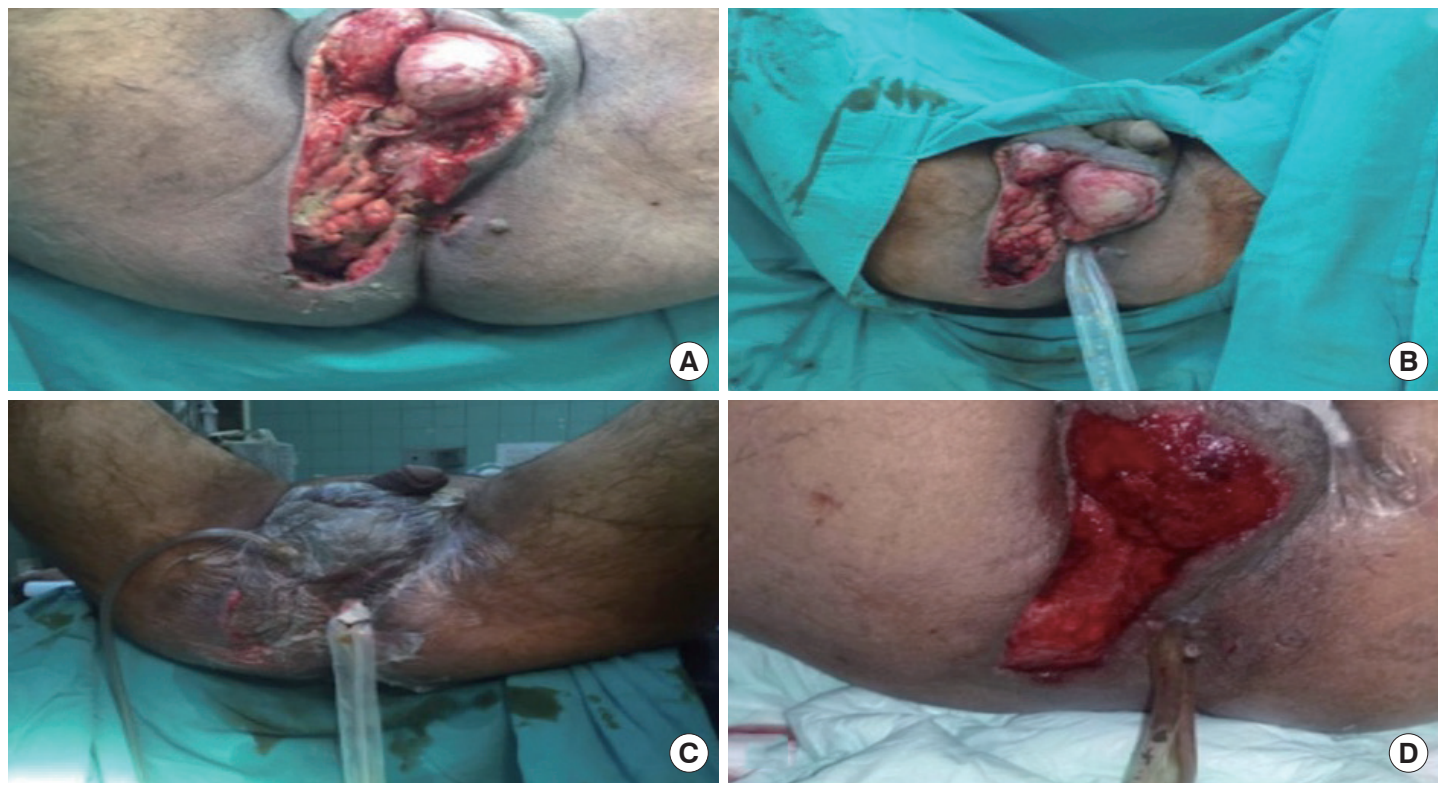

Fig. 1. Fournier gangrene case in which vacuum-assisted closure and bowel management catheter are used together. (A) An image after debridement. (B) Bowel management catheter applied. (C) Vacuum-assisted closure applied to after bowel management catheter. (D) Granulation tissue formed after vacuum-assisted closure therapy.

\section{Statistical analyses}

The variables' compatibility to normal distribution was examined using one of the analytical methods. The Spearman correlation test analyzed the correlation between continuous variables. The chi-square test, Student t-test, Mann Whitney U-test were used to compare the groups. Possible alternative cutoff points for the FGSI and CRP ratio were evaluated using area under the receiver operating characteristic (ROC) curve statistics. The prognostic ability of the clinical parameters was evaluated in both univariate and multivariable logistic regression models. Multivariate logistic regression analysis was performed to determine significant predictor variables. The model was performed with variables which found to be significant at the level of $\mathrm{P}<0.25$ in univariate analysis by stepwise backward method. Categorical measurements were summarized as numbers and percentages, and continuous measurements as mean deviation and range. The results were reported as mean standard deviation, median, number, and percentage. A P-value of $<0.05$ was considered significant. The data were analyzed with IBM SPSS Statistics for Windows ver. 24.0 (IBM Corp., Armonk, NY, USA).

\section{RESULTS}

During the study period, a total of 52 patients, with a mean age of $54.27 \pm 13.4$, diagnosed with FG fulfilling the inclusion criteria were analyzed. The study cohort consisted of male predominance with 30 patients (57.7\%). Thirty-two patients had DM, and 12 patients were malignant, with the highest proportion $(41.7 \%, \mathrm{n}=5)$ of acute myelocytic leukemia. FG is originated from the anorectal region in 36 cases (69.2\%), whereas the genitourinary region was the origin in 14 cases $(26.9 \%)$. The features related to the patient demographics and surgery-related factors in terms of mortality are presented in Table 1. The mortality rate was higher in FG patients originating from anorectal vs. genitourinary region $(\mathrm{P}=0.004)$. Patients were subjected to fecal diversion through proximal diverting stoma and $\mathrm{BMC}$ with the rates of $25.0 \%$ $(\mathrm{n}=13)$ and $19.2 \%(\mathrm{n}=10)$, respectively. The mean length of hospital stay was $26.2 \pm 17$ days for all patients. Table 1 also shows the comparison of blood transfusion, FGSI, CRP level at baseline and at the 48th hour, and CRP ratio between dead and alive patients. Overall CRP ratios were $6.7 \pm 6.6$ and $1.2 \pm 0.8$ in the survivor and non-survivor groups, respectively $(\mathrm{P}=0.001)$. In addition to that, FGSI was significantly higher in the non-survivor group compared to survivor group $(8.5 \pm 2.5$ vs. $3.5 \pm 2.2, \mathrm{P}=0.001)$. The requirement of blood transfusion was lower in the survivor group $(0.8 \pm 1.4$ vs. $3.6 \pm 3.7, \mathrm{P}=0.002)$. Conventional wound dressing was applied in 28 patients, whereas VAC dressing was used in 24 patients. A lower mortality rate was recorded for patients managed with VAC dressing ( $87.5 \%$ vs. $12.5 \%, \mathrm{P}=0.030)$.

While 10 patients required reconstruction with flaps or grafts, 2 patients required secondary suturing, 10 patients did not require secondary surgery for wound closure, and mortality occurred in 14 patients during the treatment.

Results of detectable bacterial species isolated from wound culture are presented in Table 2. Among them, Escherichia coli was the most common (34.6\%), followed by Enterococcus faecum 
Annals of The role of C-reactive protein ratio in predicting mortality in patients with Fournier gangrene Coloproctology Ismail cem Eray, et al.

Table 1. Demographic and clinical features and laboratory findings according to prognosis

\begin{tabular}{|c|c|c|c|c|}
\hline Variable & Survivor group & Non-survivor group & Total & $\mathrm{P}$-value \\
\hline Age (yr) & $52.9 \pm 13.6$ & $57.9 \pm 12.8$ & $54.3 \pm 13.4$ & 0.252 \\
\hline $18-44$ & $11(84.6)$ & $2(15.4)$ & $13(25.0)$ & 0.240 \\
\hline $45-64$ & $21(75.0)$ & $7(25.0)$ & $28(53.8)$ & \\
\hline$\geq 65$ & $6(54.5)$ & $5(45.5)$ & $11(21.2)$ & \\
\hline \multicolumn{5}{|l|}{ Sex } \\
\hline Male & $23(76.7)$ & $7(23.3)$ & $30(57.7)$ & 0.496 \\
\hline Female & $15(68.2)$ & $7(31.8)$ & $22(42.3)$ & \\
\hline \multicolumn{5}{|l|}{ Etiology } \\
\hline Anorectal & $22(61.1)$ & $14(38.9)$ & $36(69.2)$ & 0.004 \\
\hline Genitourinary & $16(100)$ & $0(0)$ & $16(30.8)$ & \\
\hline \multicolumn{5}{|l|}{ Diabetes mellitus } \\
\hline No & $17(85.0)$ & $3(15.0)$ & $20(38.5)$ & 0.125 \\
\hline Yes & $21(65.6)$ & $11(34.4)$ & $32(61.5)$ & \\
\hline \multicolumn{5}{|l|}{ Comorbidity } \\
\hline No & $17(77.3)$ & $5(22.7)$ & $22(42.3)$ & 0.559 \\
\hline Yes & $21(70.0)$ & $9(30.0)$ & $30(57.7)$ & \\
\hline \multicolumn{5}{|l|}{ Malignancy } \\
\hline No & $31(77.5)$ & $9(22.5)$ & $40(76.9)$ & 0.267 \\
\hline Yes & $7(58.3)$ & $5(41.7)$ & $12(23.1)$ & \\
\hline \multicolumn{5}{|l|}{ Debridement (time) } \\
\hline 1 & $14(70.0)$ & $6(30.0)$ & $20(38.5)$ & 0.146 \\
\hline 2 & $22(81.5)$ & $5(18.5)$ & $27(51.9)$ & \\
\hline 3 & $2(40.0)$ & $3(60.0)$ & $5(9.6)$ & \\
\hline \multicolumn{5}{|l|}{ Vacuum-assisted closure } \\
\hline No & $17(60.7)$ & $11(39.3)$ & $28(53.8)$ & 0.030 \\
\hline Yes & $21(87.5)$ & $3(12.5)$ & $24(46.2)$ & \\
\hline \multicolumn{5}{|l|}{ Bowel management catheter } \\
\hline No & $31(73.8)$ & $11(26.2)$ & $42(80.8)$ & 0.807 \\
\hline Yes & $7(70.0)$ & $3(30.0)$ & $10(19.2)$ & \\
\hline \multicolumn{5}{|l|}{ Stoma } \\
\hline No & $31(79.5)$ & $8(20.5)$ & $39(75.0)$ & 0.071 \\
\hline Yes & $7(53.8)$ & $6(46.2)$ & $13(25.0)$ & \\
\hline Hospital stay (day) & $27.6 \pm 12.1$ & $22.5 \pm 16.6$ & $26.2 \pm 17.0$ & 0.028 \\
\hline Blood transfusion (unit) & $0.8 \pm 1.4$ & $3.6 \pm 3.7$ & $1.6 \pm 2.5$ & 0.002 \\
\hline C-reactive protein ratio & $6.7 \pm 6.6$ & $1.2 \pm 0.8$ & $5.2 \pm 6.1$ & 0.001 \\
\hline Pre-CRP, baseline & $19.4 \pm 17.0$ & $12.1 \pm 10.1$ & $16.9 \pm 15.1$ & 0.426 \\
\hline Post-CRP, at the 48th hour & $4.7 \pm 3.5$ & $10.0 \pm 7.6$ & $6.5 \pm 5.7$ & 0.029 \\
\hline Fournier Gangrene Severity Index & $3.5 \pm 2.2$ & $8.5 \pm 2.5$ & $4.8 \pm 3.2$ & 0.001 \\
\hline
\end{tabular}

Values are presented as number (\%) or mean \pm standard deviation.

$(28.8 \%)$.

A moderately negative correlation was detected between FGSI and CRP ratio $(\mathrm{r}=-0.51)$. ROC analysis revealed that the cutoff FGSI value of mortality with the best discriminative ability of mortality was 5.0 with a sensitivity of $86 \%$ and a specificity of $81 \%$; the area under the ROC curve was estimated at 0.92 . The area under the ROC for CRP ratio was 0.90 ; the best cutoff value for mortality was 1.78 , with a sensitivity of $86 \%$ and a specificity 
of $82 \%$. Fig. 2 shows the results regarding mortality risks based on cutoff values of CRP ratio and FGSI, which is determined by ROC analysis.

The incidence of mortality for patients with CRP ratio of $\leq 1.78$ increased 26.7 fold for those with CRP ratio of $>1.78$ (95\% CI, 4.8-146.5; $\mathrm{P}=0.001)$. Also, the risk of death increased 39.6 times (95\% CI, 6.7-232.0; $\mathrm{P}=0.001)$ in those with an FGSI score of $\geq 5.0$ (Table 3).

Multivariate logistic regression analysis was performed to determine significant predictor variables. The model was performed with variables which found to be significant at the level of $\mathrm{P}<0.25$ in univariate analysis (CRP ratio, FGSI, age, sex, DM, malignancy, debridement, VAC, and stoma). Only CRP ratio and FGSI were found to be independent significant variables. According to this multivariable logistic regression model, the incidence of death for patients with CRP ratio of $\leq 1.78$ increased 10.3 fold for those

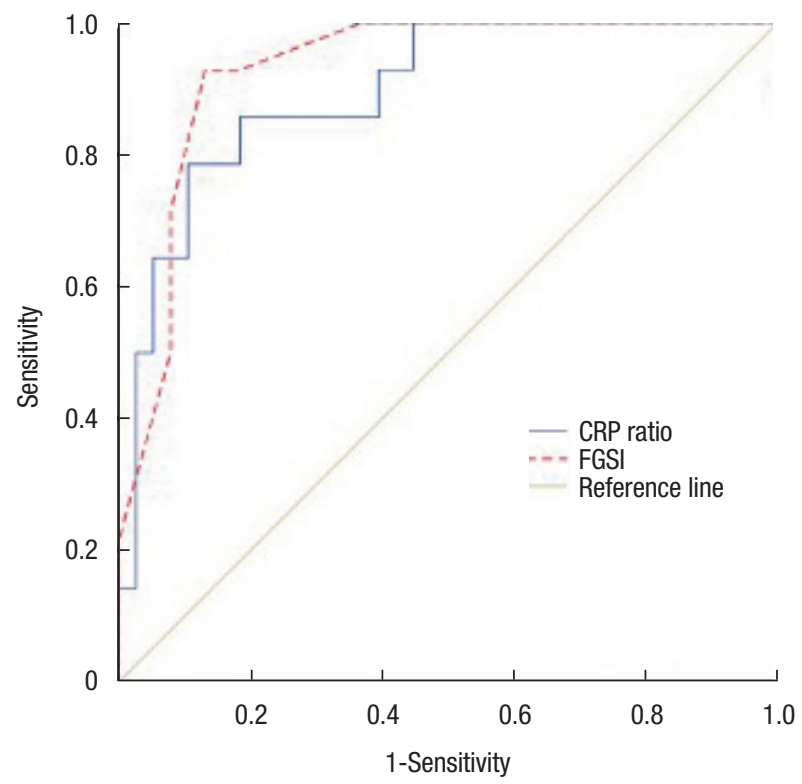

Fig. 2. Receiver operating characteristic curve of C-reactive protein (CRP) ratio and Fournier Gangrene Severe Index (FGSI). with CRP ratio of $>1.78$ ( $95 \% \mathrm{CI}, 1.5-72.2 ; \mathrm{P}=0.019)$. Also, the risk of death increased 17.8 times $(95 \% \mathrm{CI}, 2.6-121.1 ; \mathrm{P}=0.003$ ) in those with an FGSI score of $\geq 5.0$ (Table 4 ).

\section{DISCUSSION}

This study was conducted to evaluate whether the preoperative/ postoperative CRP ratio predicts death in FG. Based on our results, a simpler method the CRP ratio can be used to predict the mortality likewise FGSI. The FGSI described by Laor et al. [5] uses temperature, heart rate, respiratory rate, serum potassium and sodium, creatinine, bicarbonate levels, hematocrit, and white blood count to predict mortality. In the UFGSI developed by Yilmazlar et al. [6], disease dissemination status and age of the patient were added to these parameters. Both scoring systems have high predictive values for mortality. In the present study, it was investigated whether the CRP ratio could be as reliable as FGSI. Different studies have found sensitivity of $77 \%$ to $100 \%$ and specificity of $70 \%$ to $78.3 \%$ for predicting mortality for different cutoff values of the FGSI score $[5,6,9,10]$. Arora et al. [11] showed the

Table 2. Wound culture results

\begin{tabular}{lc}
\hline Bacterial organism & Data \\
\hline Escherichia coli & $18(34.6)$ \\
Enterococcus faecium & $15(28.8)$ \\
Klebsiella pneumoniae & $7(13.5)$ \\
Staphylococcus aureus & $4(7.7)$ \\
Psedomonas aeruginosa & $4(7.7)$ \\
Candida albicans & $3(5.8)$ \\
Prevotella bivia & $1(1.9)$ \\
Bacteroides stercoris & $1(1.9)$ \\
Enterobacter faecium & $1(1.9)$ \\
Streptococcus constella & $1(1.9)$ \\
No bacterial growth & $2(3.8)$ \\
Data missing & $2(3.8)$ \\
\hline
\end{tabular}

Values are presented as number (\%).

Table 3. The predictive ability of CRP ratio and FGSI by univariate analyses

\begin{tabular}{|c|c|c|c|c|c|}
\hline Variable & Survivor group & Non-survivor group & Total & OR $(95 \% \mathrm{Cl})$ & P-value \\
\hline CRP ratio & & & & $26.7(4.8-146.5)$ & \\
\hline$\leq 1.78$ & $7(36.8)$ & $12(63.2)$ & 19 (36.5) & & 0.001 \\
\hline$>1.78$ & 31 (93.9) & $2(6.1)$ & $33(63.5)$ & & \\
\hline FGSI & & & & $39.6(6.7-232.0)$ & \\
\hline$<5.0$ & 33 (94.3) & $2(5.7)$ & 35 (67.3) & & 0.001 \\
\hline$\geq 5.0$ & $5(29.4)$ & $12(70.6)$ & $17(32.7)$ & & \\
\hline
\end{tabular}

Values are presented as number (\%) unless otherwise specified.

CRP, C-reactive protein; FGSI, Fournier Gangrene Severity Index; OR, odds ratio; $\mathrm{Cl}$, confidence interval. 
Table 4. Results of multivariable logistic regression models

\begin{tabular}{lcccc}
\hline Variable & $\beta$ & SE & OR (95\% Cl) & P-value \\
\hline Step 1 & & & & \\
Age & 1.747 & 1.835 & $5.7(0.2-209.4)$ & 0.341 \\
Female sex & 2.688 & 1.959 & $14.7(0.3-683.3)$ & 0.170 \\
Debridement & 1.07 & 1.867 & $2.9(0.1-113.2)$ & 0.566 \\
VAC & 1.543 & 1.587 & $4.7(0.2-105.0)$ & 0.331 \\
Stoma & 3.527 & 2.496 & $34.0(0.3-4,531.1)$ & 0.158 \\
Malignancy & 0.659 & 2.007 & $1.9(0.0-98.8)$ & 0.743 \\
Diabetes mellitus & 3.384 & 2.372 & $29.5(0.3-3,081.5)$ & 0.154 \\
CRP ratio, $\leq 1.78$ & 4.475 & 1.925 & $87.8(2.0-3,818.5)$ & 0.02 \\
FGSI, $\geq 5.0$ & 3.055 & 1.845 & $21.2(0.6-790.1)$ & 0.098 \\
Constant & -9.748 & 4.558 & 0.032 & 0.032 \\
Last step & & & & 0.019 \\
CRP ratio, $\leq 1.78$ & 2.328 & 0.996 & $10.3(1.5-72.2)$ & 0.003 \\
FGSI, $\geq 5.0$ & 2.877 & 0.979 & $17.8(2.6-121.1)$ & 0.022 \\
Constant & -1.556 & 0.682 & 0.211 & \\
\hline
\end{tabular}

SE, standard error; OR, odds ratio; Cl, confidence interval; VAC, vacuum-assisted closure; CRP, C-reactive protein; FGSI, Fournier Gangrene Severity Index.

sensitivity and specificity of the FGSI to predict mortality at different cutoffs. In the present study, ROC analysis revealed that the cutoff FGSI value of mortality with the best discriminative ability of mortality was 5.0 with a sensitivity of $86 \%$ and a specificity of $81 \%$. For the CRP ratio, the best cutoff value for mortality was 1.78 , with a sensitivity of $86 \%$ and a specificity of $82 \%$. According to Laor et al. [5], a higher than 9 FGSI score was associated with a more than $75 \%$ of mortality rate. According to our result, a higher than 5.0 FGSI associated $70.6 \%$ of the risk of death, and a lower than 1.78 CRP ratio is associated with $63.2 \%$ of mortality rate. Therefore, we believe that the findings of this paper support that the CRP ratio may offer improved predictions of clinical outcomes like FGSI.

It has been reported that DM, alcohol use, immunosuppression, malignancy, and liver and kidney diseases may increase mortality $[2,12]$. Although DM is stated as the most common and most important predisposing factor for the development of mortality, this relationship is controversial. Some studies have reported that DM increases both incidence and mortality, other studies have reported that it does not increase mortality [2]. In our study, although $61.5 \%$ of the patients had DM, no significant correlation was found between DM and mortality. FG-related mortality rates in patients with FG range from $4 \%$ to $88 \%$ and have been reported to be between $20 \%$ and $40 \%$ in most cases [13]. Although higher mortality rates have been hypothesized to occur in immunocompromised FG patients, the available evidence in patients with oncohematological diseases demonstrates that FG-related mortality is in line with evidence obtained in unselected FG patients $[13,14]$. In our study, the mortality rate was found to be $26.9 \%$. Similar to the literature, malignancy was not associated with mortality during treatment of FG in our study.

Although the disease is seen in both sexes, it is more common in males. The male to female ratio was found to be 10:1 in a study of 1,726 patients [15]. However, this ratio was 1.36 in our study. Although the disease can be seen in all age groups, it has been reported that it is frequently seen in adults aged 30 to 60 years in a recent study [2]. In our study, the mean age was found to be $54.3 \pm 13.4$ years.

Wound care after debridement is very important in FG and repeated debridement may be required. In addition, the wound remains open for a long time after debridement and repetitive regular dressings are required. Even on the same day, more than one dressing may be required. Increased requirement of wound dressing has a negative impact on the patient's quality of life due to pain and pain-related discomfort after the wound dressing [16]. VAC is a wound care system that has been developed as an alternative to the standard wound care techniques, which incorporates the use of negative pressure to optimize conditions for wound healing and requires fewer painful dressing changes [16-18]. In our study, wound healing was achieved without the patients being exposed to painful dressings by making changes every 3 days in 24 patients who underwent VAC. Whether VAC treatment reduces FG mortality is controversial in the literature. Czymek et al. [17] emphasized that VAC reduces the mortality of FG from $37.5 \%$ to $5.3 \%$. However, according to different authors, it does not reduce mortality. According to our study's results, the mortality rate was lower in patients who underwent VAC $[18,19]$.

Fecal diversion may be required especially in patients when the anal sphincter complex is damaged [16, 20]. Though having an intact sphincter complex, in some cases persistent contamination 
of the wound with feces may present [1]. Preventing fecal contamination to the wound is one of the key points for healing. Contamination risk is minimized after using the VAC systems but the presence of active anal canal functions may restrict the effect of the VAC system. In such patients, BMC may be preferred instead of a surgical stoma in order to protect the wound from fecal contamination [20]. BMC is mainly used to prevent fecal contamination to the wound as in perineal burns, pressure ulcers, perineal necrotizing infections, incontinence-induced dermatitis $[21,22]$. In our study, BMC was used together with VAC in 10 patients in a compatible manner. We think that the use of BMC with or without VAC may have a positive effect on wound healing in patients with intact anal sphincter but at risk of fecal contamination. VAC therapy can increase wound healing by providing a more comfortable way than conventional wound dressing. Especially in cases where the debridement is done very close to the anal canal, the need for stomata can be avoided with BMC and it can provide more effective VAC treatment.

This study has some limitations. The insufficient sample size and retrospective nature is the main limitations of the study. Further studies need to design with a more appropriate sample size and detailed factors.

In conclusion, the mortality rate of FG is still high despite advances in treatment. FGSI is still a good scoring system for prognostic measurement, but it is complicated to use in practice. Alternatively, the CRP ratio can be simply measured and easily applied. Therefore, we recommend that CRP ratio would be used as determining the severity of FG.

\section{CONFLICT OF INTEREST}

No potential conflict of interest relevant to this article was reported.

\section{ACKNOWLEDGMENTS}

We thank Professor Doctor Gülsah Seydaoglu for her help in statistical analysis.

\section{REFERENCES}

1. Korkut M, Içöz G, Dayangaç M, Akgün E, Yeniay L, Erdoğan O, et al. Outcome analysis in patients with Fournier's gangrene: report of 45 cases. Dis Colon Rectum 2003;46:649-52.

2. Hong KS, Yi HJ, Lee RA, Kim KH, Chung SS. Prognostic factors and treatment outcomes for patients with Fournier's gangrene: a retrospective study. Int Wound J 2017;14:1352-8.

3. Sockkalingam VS, Subburayan E, Velu E, Rajashekar ST, Swamy AM. Fournier's gangrene: prospective study of 34 patients in South Indian population and treatment strategies. Pan Afr Med J 2018; 31:110.

4. Sparenborg JD, Brems JA, Wood AM, Hwang JJ, Venkatesan K.
Fournier's gangrene: a modern analysis of predictors of outcomes. Transl Androl Urol 2019;8:374-8.

5. Laor E, Palmer LS, Tolia BM, Reid RE, Winter HI. Outcome prediction in patients with Fournier's gangrene. J Urol 1995;154:8992.

6. Yilmazlar T, Ozturk E, Ozguc H, Ercan I, Vuruskan H, Oktay B. Fournier's gangrene: an analysis of 80 patients and a novel scoring system. Tech Coloproctol 2010;14:217-23.

7. Lin TY, Ou CH, Tzai TS, Tong YC, Chang CC, Cheng HL, et al. Validation and simplification of Fournier's gangrene severity index. Int J Urol 2014;21:696-701.

8. Sproston NR, Ashworth JJ. Role of C-reactive protein at sites of inflammation and infection. Front Immunol 2018;9:754.

9. Üreyen O, Acar A, Gökçelli U, Atahan MK, İlhan E. Usefulness of FGSI and UFGSI scoring systems for predicting mortality in patients with Fournier's gangrene: a multicenter study. Ulus Travma Acil Cerrahi Derg 2017;23:389-94.

10. Roghmann F, von Bodman C, Löppenberg B, Hinkel A, Palisaar J, Noldus J. Is there a need for the Fournier's gangrene severity index? Comparison of scoring systems for outcome prediction in patients with Fournier's gangrene. BJU Int 2012;110:1359-65.

11. Arora A, Rege S, Surpam S, Gothwal K, Narwade A. Predicting mortality in Fournier gangrene and validating the Fournier gangrene severity index: our experience with 50 patients in a tertiary care center in India. Urol Int 2019;102:311-8.

12. Morpurgo E, Galandiuk S. Fournier's gangrene. Surg Clin North Am 2002;82:1213-24.

13. Creta M, Longo N, Arcaniolo D, Giannella R, Cai T, Cicalese A, et al. Hyperbaric oxygen therapy reduces mortality in patients with Fournier's Gangrene. Results from a multi-institutional observational study. Minerva Urol Nefrol 2020;72:223-8.

14. Creta M, Sica A, Napolitano L, Celentano G, La Rocca R, Capece $\mathrm{M}$, et al. Fournier's gangrene in patients with oncohematological diseases: a systematic review of published cases. Healthcare (Basel) 2021;9:1123.

15. Eke N. Fournier's gangrene: a review of 1726 cases. Br J Surg 2000; 87:718-28.

16. Ozturk E, Ozguc H, Yilmazlar T. The use of vacuum assisted closure therapy in the management of Fournier's gangrene. Am J Surg 2009;197:660-5.

17. Czymek R, Schmidt A, Eckmann C, Bouchard R, Wulff B, Laubert $\mathrm{T}$, et al. Fournier's gangrene: vacuum-assisted closure versus conventional dressings. Am J Surg 2009;197:168-76.

18. Gul MO, Sunamak O, Kina U, Gunay E, Akyuz C. Fournier's gangrene: our five-year series and the role of vacuum-assisted closure in the treatment. Niger J Clin Pract 2021;24:1277-82.

19. Yanaral F, Balci C, Ozgor F, Simsek A, Onuk O, Aydin M, et al. Comparison of conventional dressings and vacuum-assisted closure in the wound therapy of Fournier's gangrene. Arch Ital Urol Androl 2017;89:208-11.

20. Eray IC, Alabaz O, Akcam AT, Ulku A, Parsak CK, Sakman G, et al. Comparison of diverting colostomy and bowel management 


\section{Coloproctology Ismail cem Eray, et al.}

catheter applications in Fournier gangrene cases requiring fecal diversion. Indian J Surg 2015;77(Suppl 2):438-41.

21. Padmanabhan A, Stern M, Wishin J, Mangino M, Richey K, DeSane M, et al. Clinical evaluation of a flexible fecal incontinence management system. Am J Crit Care 2007;16:384-93.
22. Kement M, Acar HA, Barlas IS, Aksakal N, Gezen C, Düzci U, et al. Clinical evaluation of a temporary fecal containment device for non-surgical fecal diversion in perineal burns. Ulus Travma Acil Cerrahi Derg 2011;17:123-7. 